[Article]

\title{
磺酸功能化金属-有机骨架吸附脱氮性能
}

\author{
王朝阳李 钢 $^{*}$ 孙志国 \\ (大连理工大学化工学院, 精细化工国家重点实验室, 辽宁大连 116024)
}

\begin{abstract}
摘要: 以硝基甲烷为溶剂, 采用三氟甲磺酸䣶 $\left(\mathrm{Tf}_{2} \mathrm{O}\right)$ 和浓硫酸对金属有机骨架材料 $\mathrm{MIL}-101(\mathrm{Cr})\left\{\mathrm{Cr}_{3} \mathrm{~F}\left(\mathrm{H}_{2} \mathrm{O}\right)_{2} \mathrm{O}\right.$ $\left.\left[\left(\mathrm{O}_{2} \mathrm{C}\right)-\mathrm{C}_{6} \mathrm{H}_{4}-\left(\mathrm{CO}_{2}\right)\right]_{3} \cdot n \mathrm{H}_{2} \mathrm{O}(n \sim 25)\right\}$ 进行磺酸功能化修饰, 使其孔壁配体上形成磺酸基团. 通过改变 MIL-101 $(\mathrm{Cr}) 、 \mathrm{Tf}_{2} \mathrm{O}$ 和浓硫酸的摩尔配比, 得到含有不同磺酸基团数量的 S-MIL-101(Cr), 对磺化后的材料进行了 X射线 衍射(XRD)、傅里叶变换红外(FTIR)、氮气物理吸附、酸碱电位滴定以及热重分析(TGA)表征. 结果表明, 磺酸功 能化后 MIL-101(Cr)的孔道结构仍然保持, 比表面积和孔径有所下降, 表面磺酸基团的数量根据磺化程度的不 同从 0.21 到 $0.42 \mathrm{mmol} \cdot \mathrm{g}^{-1}$ 不等. 将磺酸功能化后的 MIL-101(Cr)用于液体燃料的吸附脱氮, 发现磺酸功能化能 够增强 MIL-101(Cr)与含氮化合物的相互作用, 有利于其对碱性氮化物的吸附脱除. 相对于未经磺化的样品, 按 照摩尔配比 $n(\mathrm{MIL}-101(\mathrm{Cr})): n\left(\mathrm{H}_{2} \mathrm{SO}_{4}\right): n\left(\mathrm{Tf}_{2} \mathrm{O}\right)=1: 3: 4.5$ 反应得到的磺酸功能化 MIL-101(Cr)对喹啉和呫哚的吸 附量提高较大, 其对喹啉和吲哚的 Langmuir 最大吸附量分别提高了 $12.2 \%$ 和 $6.3 \%$. 通过乙醇洗涤, 吸附剂可 再生, 经过三次再生之后的吸附剂对模拟燃料中含氮化合物的吸附量没有明显的降低.
\end{abstract}

关键词：金属有机骨架; 磺酸功能化; 吸附脱氮; Langmuir吸附; 吸附等温线 中图分类号: 0647

\section{Denitrogenation through Adsorption to Sulfonated Metal-Organic Frameworks}

\author{
WANG Zhao-Yang LI Gang* SUN Zhi-Guo \\ (State Key Laboratory of Fine Chemicals, School of Chemical Engineering, Dalian University of Technology, \\ Dalian 116024, Liaoning Province, P. R. China)
}

\begin{abstract}
Sulfonic acid functionalized MIL-101(Cr) (S-MIL-101(Cr)) are obtained by sulfonation of MIL$101(\mathrm{Cr})\left(\mathrm{Cr}_{3} \mathrm{~F}\left(\mathrm{H}_{2} \mathrm{O}\right)_{2} \mathrm{O}\left[\left(\mathrm{O}_{2} \mathrm{C}\right)-\mathrm{C}_{6} \mathrm{H}_{4}-\left(\mathrm{CO}_{2}\right)\right]_{3} \cdot n \mathrm{H}_{2} \mathrm{O}(n \sim 25)\right)$ using triflic anhydride and sulfuric acid. The amount of sulfonic groups in the framework can be controlled by changing the molar ratio of MIL-101(Cr), triflic anhydride, and sulfuric acid. The sulfonated samples were characterized by X-ray diffraction (XRD), Fourier transform infrared (FTIR) spectroscopy, nitrogen physical adsorption/desorption, acid- base potentiometric titration, and thermogravimetric analysis (TGA). The results show that the sulfonated samples retain the general structure of MIL-101(Cr), but the specific areas and pore diameters decrease. The sulfonated samples formed with between 0.21 and $0.42 \mathrm{mmol} \cdot \mathrm{g}^{-1}$ of sulfonic acid groups. The adsorptive denitrogenation of a model fuel by different S-MIL-101(Cr) samples was investigated in batch adsorption experiments. Sulfonation can strengthen the interactions between nitrogen-containing compounds (NCCs) and the adsorbent. The sample obtained using a molar ratio of $n(\mathrm{MIL}-101(\mathrm{Cr})): n\left(\mathrm{H}_{2} \mathrm{SO}_{4}\right): n\left(\mathrm{Tf}_{2} \mathrm{O}\right)=1: 3: 4.5$ had the largest adsorption capacity for quinoline and indole. Compared with the bare MIL-101(Cr), this sulfonated material showed enhancement of the maximum adsorption capacity by $12.2 \%$ and $6.3 \%$ for quinoline and indole, respectively. Regeneration of the used adsorbent was conducted by washing with ethanol, and the adsorptive capacity for NCCs from the model-fuel showed no obvious decrease after three cycles of use.
\end{abstract}

Received: June 12, 2013; Revised: September 2, 2013; Published on Web: September 2, 2013.

*Corresponding author. Email: liganghg@dlut.edu.cn; Tel: +86-411-84986113.

The project was supported by the National Key Basic Research Program of China (973) (2011CB201301).

国家重点基础研究发展计划项目(973) (2011CB201301)资助 
Key Words: Metal-organic framework; Sulfoacid functionalization; Adsorptive denitrogenation; Langmuir adsorption; Adsorption isothermal

\section{1 引 言}

油品中的含氮化合物是大气污染的来源之一, 会对人类的生存环境造成重大危害, 而且这些含氮 化合物在油品的催化加工过程中能强烈地吸附在 催化剂表面, 占据活性位, 造成催化剂中毒失活. ${ }^{1,2}$ 另外, 油品中含氮化合物会腐蚀炼油过程中管道、 泵等设备. 含氮化合物的存在, 还会使油品的安定 性变差, 储存时容易受到光、空气中的氧气以及金 属等的催化作用而生成胶质, 进而使油品颜色变 深, 生成沉淀等, 影响油品品质. 我国原油的特点之 一就是含氮量偏高, 因此对于氮化物的脱除显得尤 为必要. ${ }^{3}$

传统工艺中, 大多是在高温(573-653 K)和高压 (2-10 MPa)条件下采用催化加氢处理的方式来脱 除油品中的含氮化合物. ${ }^{4-6}$ 加氢脱氮可以有效脱除 油品中的脂肪胺、苯胺类含氮化合物, 然而一些杂 环含氮化合物因其加氢反应活性较低, 采用加氢方 法很难将其脱除. 因此, 许多新的油品脱氮工艺, 例 如吸附脱氮 $(A D N)$ 、氧化脱氮 $(O D N)$ 以及酸萃取脱 氮, 得到人们的广泛关注. 其中, 采用多孔材料吸附 脱氮, 因其操作条件较为温和、环境友好, 受到众多 研究者的青睐. 目前已经报道的吸附脱氮材料包括 活性炭, 89 金属氧化物或金属硫化物, ${ }^{10,11}$ 分子篮类材 料 ${ }^{2}$ 等. 然而, 这些材料对氮化物的吸附量普遍较 低, 例如 Laredo 等 ${ }^{13}$ 报道的 $\mathrm{Cu}^{+} \mathrm{Y}$ 沸石对氮化物的吸 附量仅为 $3 \mathrm{mg} \cdot \mathrm{g}^{-1}$ (以氮原子计), Almarri等 ${ }^{8}$ 制备的 活性炭对喹啉的吸附量为 $17.64 \mathrm{mg} \cdot \mathrm{g}^{-1}$. 因此, 寻找 或设计一种具有较大氮化物吸附容量的吸附剂对 于吸附脱氮工艺的开发至关重要.

金属-有机骨架(MOFs)是一类新型的多孔固体 材料, 它们是由无机金属离子或离子簇与有机配体 通过配位键桥联而成. 这类材料因其具有比表面积 较大、孔隙率较高、孔道形状和大小可调以及孔壁 上的配体可功能化等优点, ${ }^{14}$ 受到人们的广泛关注. 利用这类材料在气相以及液相吸附分离领域的研 究已经有不少报道, 其中包括吸附脱硫(ADS) ${ }^{15-17}$ 和 吸附脱氮..$^{8,19}$ Maes 等 ${ }^{18}$ 采用一种具有介孔结构的 MOF 材料 MIL-100(Fe) 对模拟燃料中的含氮化合物 进行吸附脱除, 结果表明 MIL-100(Fe)具有良好的吸 附脱氮性能, 可以将初始含氮化合物浓度为 0.15 $\mathrm{mol} \cdot \mathrm{L}^{-1}$ 的模拟燃料(庚烷)中 $36 \%$ 的吲哚吸附脱除, 展示了 MOF 材料对液体燃料中含氮化合物较高的 吸附容量. 金属-有机骨架材料最显著的特点之一就 是其孔道结构中的有机配体容易通过化学方法进 行修饰, 特别是可通过合成后改性的方法(PSM) 向 MOFs 的孔道中引入功能性基团, 以达到提高吸附 容量的目的. 这种方法在 MOFs 的研究领域已经被 广泛报道. ${ }^{20}$

在本文利用磺化反应, 在硝基甲烷溶剂中, 通 过三氟甲磺酸䣶 $\left(\mathrm{Tf}_{2} \mathrm{O}\right)$ 和浓硫酸对 MIL-101(Cr) $\left(\mathrm{Cr}_{3} \mathrm{~F}\left(\mathrm{H}_{2} \mathrm{O}\right)_{2} \mathrm{O}\left[\left(\mathrm{O}_{2} \mathrm{C}\right)-\mathrm{C}_{6} \mathrm{H}_{4}-\left(\mathrm{CO}_{2}\right)\right]_{3} \cdot n \mathrm{H}_{2} \mathrm{O}(n \sim 25)\right)$ 进行 磺酸功能化修饰, 从而得到含有不同磺酸基团数量 的 MIL-101(Cr), 并将这些材料用于模拟燃料吸附脱 氮性能, 探讨了其对含氮化合物的吸附作用机理.

\section{2 实验部分}

\section{$2.1 \mathrm{MIL}-101(\mathrm{Cr})$ 的合成}

MIL-101(Cr) 的合成参照文献, ${ }^{21}$ 将九水硝酸铬 $(99 \%)$ 、对苯二甲酸 $(99 \%)$ 、去离子水、氢氟酸 $40 \%$ 的 水溶液)按照摩尔比 1:1:270:2.5 混合, 在室温下摚拌 均匀, 装入 $100 \mathrm{~mL}$ 晶化釜, 在 $493 \mathrm{~K}$ 的温度下反应 $8 \mathrm{~h}$, 自然冷却至室温过滤, 然后分别用乙醇和 0.2 $\mathrm{mol} \cdot \mathrm{L}^{-1}$ 的 $\mathrm{NH}_{4} \mathrm{~F}$ 溶液纯化, 于 $333 \mathrm{~K}$ 真空干燥 $12 \mathrm{~h}$.

\subsection{MIL-101(Cr) 的磺酸功能化}

MIL-101(Cr) 的磺酸功能化参考文献, ${ }^{22}$ 将真空 干燥后的 MIL-101(Cr)、 $\mathrm{Tf}_{2} \mathrm{O}\left(98 \%\right.$, Aladdin) 和 $\mathrm{H}_{2} \mathrm{SO}_{4}$ (98\%)分别按照表 1 给出的摩尔配比及反应条件进 行反应. 反应完成之后, 将产物过滤, 用去离子水和

表 1 S-MIL-101(Cr)的制备过程

Table 1 Preparation procedure for S-MIL-101(Cr)

\begin{tabular}{ccccc}
\hline Material & $\begin{array}{c}n(\mathrm{MIL}-101(\mathrm{Cr})): \\
n\left(\mathrm{H}_{2} \mathrm{SO}_{4}\right): n\left(\mathrm{Tf}_{2} \mathrm{O}\right)^{\mathrm{a}}\end{array}$ & Solvent & $T /{ }^{\circ} \mathrm{C}$ & $t / \mathrm{h}$ \\
\hline S1-MIL-101(Cr) & $1: 1: 1.5$ & $\begin{array}{c}\mathrm{CH}_{3} \mathrm{NO}_{2} \\
\left(100 \mathrm{~mL} \cdot \mathrm{g}^{-1}\right)\end{array}$ & 30 & 1 \\
S2-MIL-101(Cr) & $1: 3: 4.5$ & $\begin{array}{c}\mathrm{CH}_{3} \mathrm{NO}_{2} \\
\left(100 \mathrm{~mL} \cdot \mathrm{g}^{-1}\right)\end{array}$ & 30 & 3 \\
S3-MIL-101(Cr) & $1: 5: 7.5$ & $\begin{array}{c}\mathrm{CH}_{3} \mathrm{NO}_{2} \\
\left(100 \mathrm{~mL} \cdot \mathrm{g}^{-1}\right)\end{array}$ & 30 & 5 \\
\hline
\end{tabular}

MIL-101(Cr): $\left(\mathrm{Cr}_{3} \mathrm{~F}\left(\mathrm{H}_{2} \mathrm{O}\right)_{2} \mathrm{O}\left[\left(\mathrm{O}_{2} \mathrm{C}\right)-\mathrm{C}_{6} \mathrm{H}_{4}-\left(\mathrm{CO}_{2}\right)\right]_{3} \cdot n \mathrm{H}_{2} \mathrm{O}(n \sim 25)\right)$; $\mathrm{Tf}_{2} \mathrm{O}$ : triflic anhydride; S-MIL-101(Cr): sulfoacid functionalized MIL$101(\mathrm{Cr}) .{ }^{a}$ molar ratio of the reactants 
丙酮进行充分洗涤, 然后将得到的样品分散到 343 $\mathrm{K}$ 的乙醇中洗涤 $24 \mathrm{~h}$, 之后烘干即可.

\section{3 材料表征}

采用 Rigaku D/Max 2400 型衍射仪对材料进行 $\mathrm{X}$ 射线衍射 $(\mathrm{XRD})$ 测试, 使用 $\mathrm{Cu}-K_{\alpha}(\lambda=0.154184 \mathrm{~nm})$ 射线源, 管电压 $40 \mathrm{kV}$, 管电流 $40 \mathrm{~mA}$, 扫描速率 $0.02\left(^{\circ}\right) \cdot \mathrm{min}^{-1}$, 扫描区间 $2 \theta=2^{\circ}-20^{\circ}$. 傅里叶变换红 外(FTIR)光谱在 Bruker EQUINOX55 红外分光计上 进行, 样品测试之前采用 $\mathrm{KBr}$ 压片. 氮气物理吸附 实验通过北京精微高博公司生产的 JW-BK122W 型 物理吸附仪进行, 测试温度为液氮温度 $(-77 \mathrm{~K})$, 样 品在测试之前 $423 \mathrm{~K}$ 真空处理 $3 \mathrm{~h}$. 比表面积采用 Brunner-Emmett-Teller (BET)法测得, 孔径分布通过 吸附等温线采用 Barrett-Joyner-Halenda (BJH) 法测 得. 采用酸-碱电位滴定测试磺化后样品的磺酸基团 数量. ${ }^{23}$ 准确称取 $0.5 \mathrm{~g}$ 经过真空干燥的样品, 将其分 散在 $20 \mathrm{~mL}$ 饱和 $\mathrm{NaCl}$ 溶液中, 然后室温摚拌 $12 \mathrm{~h}$ 左 右, 过滤后采用 $0.01 \mathrm{~mol} \cdot \mathrm{L}^{-1}$ 的 $\mathrm{NaOH}$ 溶液对滤液进 行酸-碱电位滴定, 用上海辰华 CHI-604D 电化学工 作站测量滴定过程中溶液开路电位变化. 热重分析 (TGA) 在 METTLER TOLEDO 公司的 TGA/SD$\mathrm{TA} 851^{\mathrm{e}}$ 型热重分析仪上进行, 空气氛围, 温度范围 303-873 K, 升温速率 $10 \mathrm{~K} \cdot \mathrm{min}^{-1}$.

\section{4 间歇吸附实验}

吸附实验采用间歇吸附. 首先, 将含氮化合物 (碱性氮化物喹啉(99\%), 非碱性氮化物弜哚(98.5\%)) 分别溶解在正辛烷(99\%)中, 配制成一系列不同氮 含量 $\left(50-1000 \mu \mathrm{g} \cdot \mathrm{g}^{-1}\right)$ 的模拟燃料. 在吸附实验之前 采用 $423 \mathrm{~K}$ 真空干燥吸附剂 $12 \mathrm{~h}$, 每次吸附实验按 照一定的剂油质量比称取一定量的吸附剂加入模 拟燃料中充分搅拌 $120 \mathrm{~min}$, 采用水浴加热维持吸附 温度的恒定. 最后采用离心方式分离吸附剂和燃 料. 采用 KY-3000N 型化学发光定氮仪(江苏科苑电 子仪器有限公司)测定反应前后样品中的氮含量.

以吸附量 $q$ 和脱除率 $r$ 作为评价吸附剂对不同 含氮化合物的吸附能力的指标, 它们的计算方法分 别如下:

$$
\begin{gathered}
q=\frac{m_{\mathrm{f}}\left(C_{0}-C_{\mathrm{e}}\right)}{m_{\mathrm{a}}} \times 10^{-3} \\
r=\frac{\left(C_{0}-C_{\mathrm{e}}\right)}{C_{0}} \times 100 \%
\end{gathered}
$$

上式中: $q$ 为单位质量吸附剂上吸附的氮原子的质 量, 单位为 $\mathrm{mg} \cdot \mathrm{g}^{-1} ; C_{0}$ 为吸附前模拟燃料中氮原子
的初始浓度, 单位为 $\mu \mathrm{g} \cdot \mathrm{g}^{-1} ; C_{\mathrm{e}}$ 为吸附平衡后模拟燃 料中氮原子的浓度, 单位为 $\mu \mathrm{g} \cdot \mathrm{g}^{-1} ; m_{\mathrm{a}}$ 为吸附剂的质 量, 单位为 $\mathrm{g} ; m_{\mathrm{f}}$ 为模拟燃料的质量, 单位为 $\mathrm{g} ; r$ 为脱 除率.

\section{3 结果与讨论}

\section{1 材料的表征}

将所合成出的材料的 XRD 谱图与原文献的 谱图 ${ }^{21}$ 对比, 可以确定所合成出的材料具有 MIL101(Cr) 的结构. 与未经磺化的 MIL-101(Cr) 的谱图 对比, 经过磺化反应后的 MIL-101(Cr)仍保留了其基 本结构(图 1). 随着反应原料配比(表 1)中 $\mathrm{Tf}_{2} \mathrm{O}$ 以及 浓硫酸用量的相对增加以及反应时间的延长, 所得 到的磺化后材料的结晶度逐渐降低, 说明材料的结 构遭到了不同程度的破坏. 其中, S3-MIL-101(Cr)被 破坏的程度最大, 说明 MIL-101(Cr) 在此反应体系 下骨架结构已经不能稳定存在.

通过红外谱图的研究, 可以证实所得到的磺化 材料中含有磺酸基团. 图 2 给出了这些经过磺化的 MIL-101(Cr)与未经磺化的 MIL-101(Cr) 的 FTIR 谱 图, 对比波数在 2000 到 $800 \mathrm{~cm}^{-1}$ 区域内的谱图可以 发现, 在 $1260 \mathrm{~cm}^{-1}$ 左右处有明显的新谱带峰出现, 可以归属为磺酸基团中 $\mathrm{O}=\mathrm{S}=\mathrm{O}$ 的对称伸缩振动; 而在波数 $1430 \mathrm{~cm}^{-1}$ 可以看到一个新的肩峰的存在, 使得 MIL-101(Cr)在此范围内的峰明显宽化了, 这应 该是磺酸基团中 $\mathrm{O}=\mathrm{S}=\mathrm{O}$ 的不对称伸缩振动所形 成的吸收峰..$^{24,25}$ 在波数为 $1100 \mathrm{~cm}^{-1}$ 处的峰可以归属 为苯环上的氢被磺酸基取代后的面内骨架振动吸 收峰, 波数 $1030 \mathrm{~cm}^{-1}$ 处的吸收峰则归属为 $\mathrm{S}-\mathrm{O}$ 伸 缩振动. ${ }^{26}$ 这些峰的存在说明磺化后的 MIL-101(Cr)

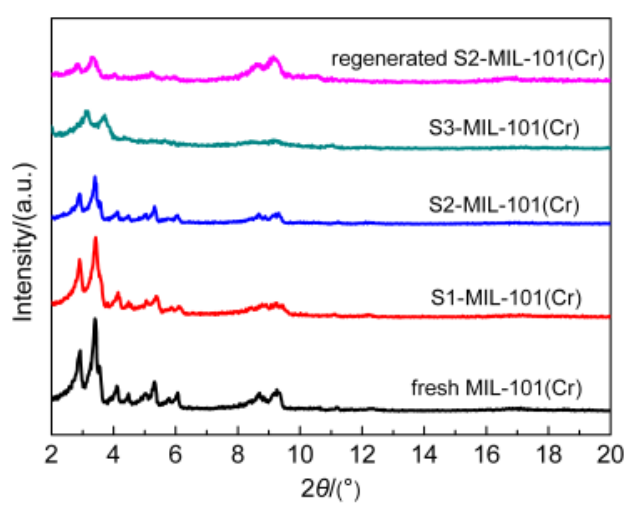

图 1 不同磺化程度的 MIL-101(Cr)样品的 XRD 谱图 Fig.1 XRD patterns of different sulfated MIL-101(Cr) samples 


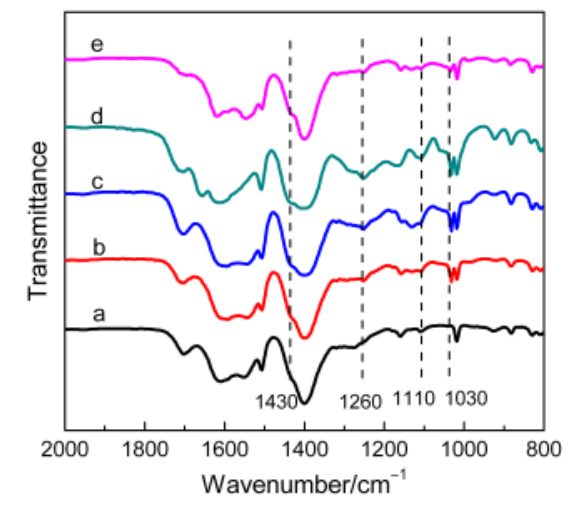

图 2 不同磺化程度的 MIL-101(Cr)样品的 FTIR 谱图

Fig.2 FTIR spectra of different sulfated MIL-101(Cr) samples

(a) MIL-101(Cr); (b) S1-MIL-101(Cr); (c) S2-MIL-101(Cr);

(d) S3-MIL-101(Cr); (e) regenerated S2-MIL-101(Cr)

的结构中的确有磺酸基团存在.

图 3 和表 2 分别给出了经过磺化的 MIL-101(Cr) 与未经磺化的 MIL-101(Cr)的氮气物理吸附-脱附等 温线和结构参数, 可以发现经过磺化反应后 MIL101(Cr)的 BET 比表面积和孔容均有明显下降, BJH 孔径也有所下降. 这一方面由于磺酸基团进入孔道 引起, 另一方面由于 MIL-101(Cr)在反应过程中骨架 结构的破坏. 从吸附等温线的形状来看, S2-MIL101(Cr)和 S1-MIL-101(Cr) 仍然和未磺化的样品保 持一致, 而 S3-MIL-101(Cr) 则变化较大, 这也说明 S3-MIL-101(Cr)的基本结构被破坏的程度最大. 另 外, 与未经磺化的 MIL-101(Cr) 的吸附等温线相比, S1-MIL-101(Cr)和 S2-MIL-101(Cr) 在比压为 0.2 左 右处突跃的程度降低, 表明磺化反应一定程度上降 低了MIL-101(Cr) 孔结构有序性. 通过酸碱电位滴 定, 测得磺化之后的不同样品所含有的磺酸基团的 数量(表 2). 可以发现样品中所含有的磺酸基团的数

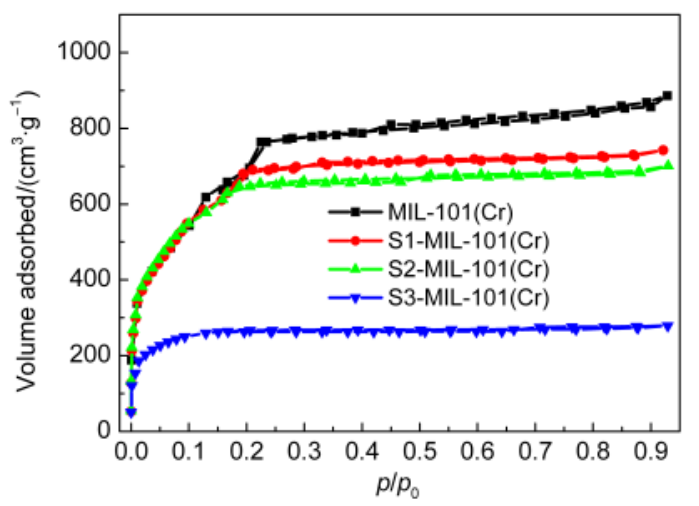

图 3 不同磺化程度的 MIL-101(Cr)样品的氮气 吸附-脱附等温线

Fig.3 Nitrogen adsorption-desorption isothermals of different sulfated MIL-101(Cr) samples
表 2 不同吸附剂的物理结构以及磺酸根的量

Table 2 Texture and chemical properties of different adsorbents

\begin{tabular}{lcccc}
\hline \multicolumn{1}{c}{ Adsorbent } & $S_{\mathrm{BET}} /\left(\mathrm{m}^{2} \cdot \mathrm{g}^{-1}\right)$ & $V_{\text {porr }} /\left(\mathrm{cm}^{3} \cdot \mathrm{g}^{-1}\right)$ & $A_{\mathrm{BJH}} / \mathrm{nm}$ & $n_{\text {sulf }} /\left(\mathrm{mmol} \cdot \mathrm{g}^{-1}\right)$ \\
\hline MIL-101(Cr) & 2642 & 1.23 & 2.31 & 0 \\
S1-MIL-101(Cr) & 2333 & 1.15 & 2.20 & 0.21 \\
S2-MIL-101(Cr) & 2158 & 1.08 & 2.09 & 0.33 \\
S3-MIL-101(Cr) & 869 & 0.43 & 1.98 & 0.42 \\
\hline
\end{tabular}

$S_{\text {BET: }}$ BET specific surface area; $V_{\text {pore }}$ pore volume; $A_{\text {ВJH }}$ : aperture; $n_{\text {sulf: }}$ the amount of sulfonic acid group

量从 S1-MIL-101(Cr) 到 S3-MIL-101(Cr) 逐渐增加. 虽然 S3-MIL-101(Cr) 的基本结构遭到很大程度破 坏, 但其所含有的磺酸基团的数量却是最多的.

图 4 给出了磺化后样品 S2-MIL-101(Cr)的热重 分析曲线, 由图中可以看出在考察的温度范围内大 致有两步失重: 第一步发生在 303-373 K之间, 对应 于物理水的失去; 第二步发生在 600-660 K之间, 对 应于样品骨架的分解. 可以看出, 材料具有较好的 热稳定性, 足以满足吸附脱氮应用的要求.

\section{2 模拟燃料吸附脱氮}

分别采用 S1-MIL-101(Cr)、S2-MIL-101(Cr)、S3MIL-101(Cr) 为吸附剂, 对以正辛烷为溶剂的模拟燃 料中的喹啉和吲哚进行吸附, 得到了如图 5 和图 6 所示的吸附等温线. 对比不同磺化度样品对喹啉的 吸附量, 由图 5 可以发现, 虽然 S2-MIL-101(Cr) 和 S1-MIL-101(Cr)的 BET 比表面积和孔容有一定程度 的下降, 但磺化后的样品 S2-MIL-101(Cr) 和 S1-MIL101(Cr) 对喹啉的吸附量有所提高. 其中, S2-MIL101(Cr)在整个浓度范围内对喹啉的吸附量是所有 样品中最高的, S1-MIL-101(Cr) 稍次之. 对于 S3MIL-101(Cr), 在平衡浓度较小的区域, 其对喹啉的 吸附量高于未经磺化的 MIL-101(Cr); 然而由于其

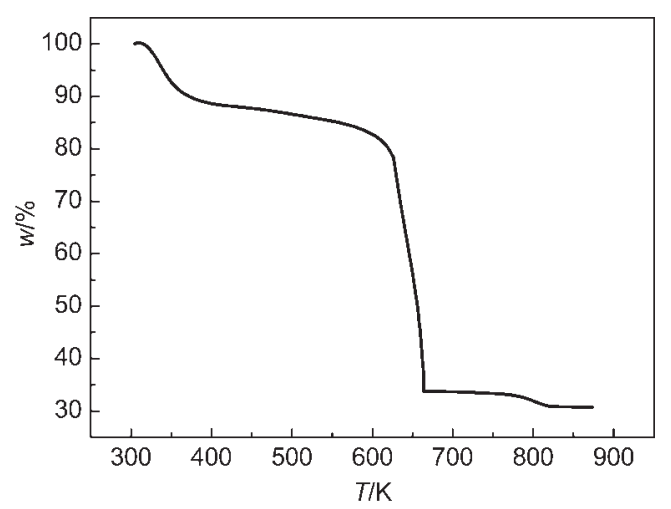

图 4 S2-MIL-101(Cr) 的热重分析曲线 Fig.4 TGA curve of S2-MIL-101(Cr) 


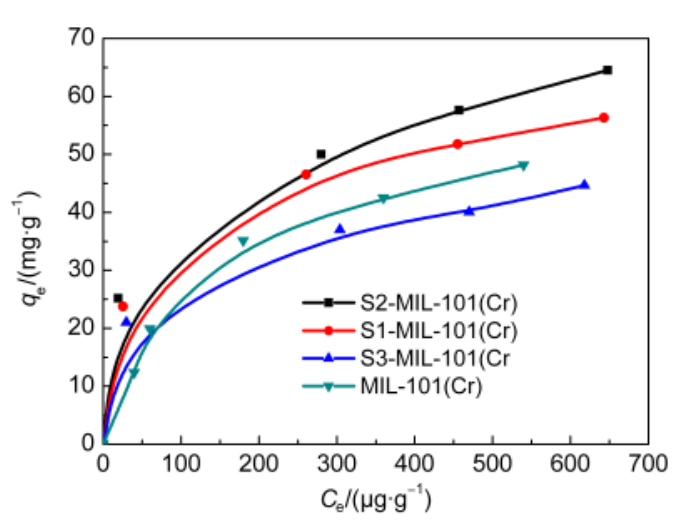

图 $5303 \mathrm{~K}$ 下不同磺化程度的 MIL-101(Cr)样品 对模拟燃料中喹啉的吸附等温线

Fig.5 Adsorption isothermals for quinoline in model fuel over different sulfated MIL-101(Cr) samples at $303 \mathrm{~K}$

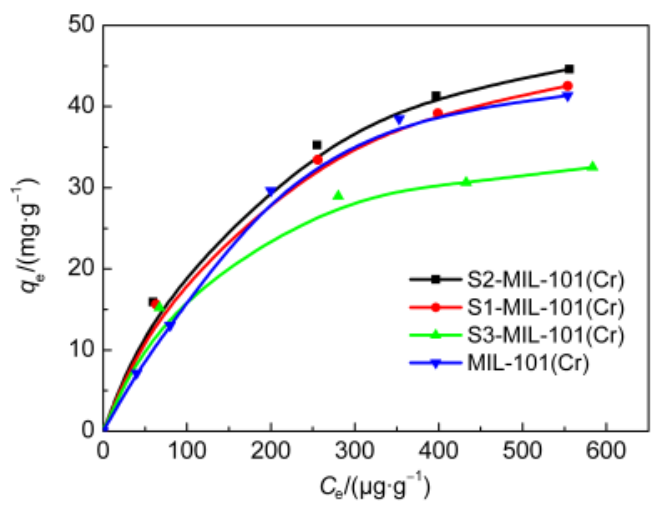

图 $6303 \mathrm{~K}$ 下不同磺化程度的 MIL-101(Cr)样品 对模拟燃料中吲哚的吸附等温线

Fig.6 Adsorption isothermals for indole in model fuel over different sulfated MIL-101(Cr) samples at $303 \mathrm{~K}$

比表面积和孔容积大大下降, 在较高平衡浓度区 域, S3-MIL-101(Cr) 对喹啉的吸附量要低于未经磺 化的样品. 吸附等温线中, 材料在低浓度区域的吸 附能力反映了吸附剂与吸附质的相互作用强弱. ${ }^{8}$ 因 此, 对比低浓度区域的吸附等温线, 可以得出磺酸 功能化 MIL-101(Cr) 能够增强其与喹啉之间的相互 作用.

图 6 为不同磺化度样品和未经磺化样品对模拟 燃料中吲哚的吸附等温线. 从图中可以看出, 磺化 后的样品 S2-MIL-101(Cr) 对吲哚的吸附量略有提 高, 但提高的程度和喹啉相比已变小, 这是因为吲 哚是中性氮化物而喹啉是碱性氮化物. 由于酸碱作 用, 磺酸基更倾向于吸附碱性氮化物. S1-MIL-101 (Cr) 对吲哚的吸附量和未磺化样品相近, 因此, 可以 认为磺酸基团的引入对于吲哚的吸附影响不大. 尽 管 S3-MIL-101(Cr)样品所含的磺酸基团数量最多, 但由于其结构遭到严重破坏, 比表面积和孔容变
表 3 不同磺化程度的 MIL-101(Cr)样品对模拟燃料中喹啉 和吲哚最大吸附量 $\left(Q_{0}\right)$ 以及 Langmuir 常数 $(b)$ 和拟合的 相关性系数 $\left(R^{2}\right)$

Table 3 The maximum adsorption capacities $\left(Q_{0}\right)$, Langmuir constant (b), and correlation coefficients $\left(R^{2}\right)$ of quinoline and indole over different sulfated MIL-101(Cr) samples

\begin{tabular}{|c|c|c|c|c|}
\hline $\begin{array}{l}\text { Nitrogen-containing } \\
\text { compound }\end{array}$ & Adsorbent & $\frac{Q_{0}}{\left(\mathrm{mg} \cdot \mathrm{g}^{-1}\right)}$ & $\frac{b}{\left(\mathrm{~L} \cdot \mathrm{mol}^{-1}\right)}$ & $R^{2}$ \\
\hline \multirow[t]{4}{*}{ quinoline } & MIL-101(Cr) & 64.69 & 37.73 & 0.996 \\
\hline & S1-MIL-101(Cr) & 66.52 & 38.38 & 0.995 \\
\hline & S2-MIL-101(Cr) & 72.58 & 42.32 & 0.984 \\
\hline & S3-MIL-101(Cr) & 46.51 & 37.93 & 0.989 \\
\hline \multirow[t]{4}{*}{ indole } & MIL-101(Cr) & 49.44 & 21.07 & 0.958 \\
\hline & S1-MIL-101(Cr) & 50.14 & 21.28 & 0.999 \\
\hline & S2-MIL-101(Cr) & 52.56 & 22.38 & 0.999 \\
\hline & S3-MIL-101(Cr) & 37.88 & 16.74 & 1.000 \\
\hline
\end{tabular}

小, 其对于吲哚的吸附量明显降低.

通过 Langmuir 方程对所得到的吸附等温线进 行拟合, 得到了不同磺化度样品和未磺化的样品分 别对于喹啉和吲哚的最大吸附容量 $Q_{0} 、$ Langmuir 常 数 $b$ 以及拟合的相关性系数 $\left(R^{2}\right)($ 表 3$)$. 首先, 从表 3 中的相关性系数可以判断所有样品对氮化物喹啉 和吲哚的吸附均属于 Langmuir型吸附. 从表中不同 样品的最大吸附量 $Q_{0}$ 的对比可以发现, 对于喹啉, S2-MIL-101 $(\mathrm{Cr})$ 具有最大的 $Q_{0}$ 值, 相对于未经磺化 的样品, $Q_{0}$ 提高了 $12.2 \%$. 而相同的样品对于吲哚, $Q_{0}$ 仅仅提高了 $6.3 \%$. 因此, 可以认为对 MIL-101(Cr) 适度磺酸功能化能够增强其与氮化物的相互作用, 提高其对氮化物的吸附容量, 并且更有利于对碱性 氮化物的吸附.

\section{3 吸附剂的再生}

吸附剂的再生性能对于其工业应用来说至关 重要. 简单的吸附剂再生工艺能够降低操作成本、 减少环境污染. 本节采用极性溶剂乙醇对吸附饱和 之后的吸附剂进行了再生处理, 具体操作方法为: 将吸附饱和后的吸附剂分散于无水乙醇中 $(200 \mathrm{~mL}$. $\left.\mathrm{g}^{-1}\right)$, 然后在室温下搅拌 $30 \mathrm{~min}$, 离心分离后置于真 空烘箱中 $423 \mathrm{~K}$ 干燥 $12 \mathrm{~h}$.

以 S2-MIL-101 $(\mathrm{Cr})$ 为例, 考察其在含喹啉的模 拟燃料 $\left(50 \mu \mathrm{g} \cdot \mathrm{g}^{-1}\right.$, 以氮原子计 $)$ 中重复使用及再生 性能. 吸附剂的重复使用结果如表 4 所示, 第 1 次和 第 2 次再生后的 S2-MIL-101(Cr) 对模拟燃料中的含 氮化合物仍然可以完全脱除, 展现出了良好的模拟 燃料吸附脱氮性能. 第 3 次再生的 S2-MIL-101(Cr) 对模拟燃料中氮化物的脱除率略有下降, 这可能与 
表 4 S2-MIL-101(Cr)对喹啉的吸附脱氮及其 再生性能评价 ${ }^{\mathrm{a}}$

Table 4 Removal of quinoline by fresh and regenerated S2-MIL-101 $(\mathrm{Cr})^{\mathrm{a}}$

\begin{tabular}{lccrc}
\hline \multicolumn{1}{c}{ Adsorbent ${ }^{\mathrm{b}}$} & $c_{\mathrm{i}} /\left(\mu \mathrm{g} \cdot \mathrm{g}^{-1}\right)$ & $c_{\mathrm{f}} /\left(\mu \mathrm{g} \cdot \mathrm{g}^{-1}\right)$ & $r / \%$ & $n_{\text {sutf }} /\left(\mathrm{mmol} \cdot \mathrm{g}^{-1}\right)$ \\
\hline MIL-101(Cr) & 50 & 4.4 & 91 & - \\
S2-MIL-101(Cr) & 50 & 0 & 100 & 0.33 \\
S2-MIL-101(Cr)-R1 & 50 & 0 & 100 & 0.31 \\
S2-MIL-101(Cr)-R2 & 50 & 0 & 100 & 0.28 \\
S2-MIL-101(Cr)-R3 & 50 & 1.6 & 97 & 0.20 \\
\hline
\end{tabular}

adsorption conditions: adsorbent amount, $20 \mathrm{~g} \cdot \mathrm{L}^{-1}$; time, $1 \mathrm{~h}$; temperature, $303 \mathrm{~K} .{ }^{b} \mathrm{R} 1, \mathrm{R} 2$, and $\mathrm{R} 3$ denote regeneration for 1, 2, and 3 times, respectively. $c_{\mathrm{i}}$ : initial concentration; $c_{\mathrm{f}}$ : final concentration;

$$
r \text { : removal rate }
$$

其表面磺酸基团的损耗有关. 再生后的吸附剂的 XRD 谱图和FTIR 谱图分别如图 1 和图 2 所示. 从图 1 中可以看出, 经过三次再生处理的 S2-MIL-101 (Cr)仍然保留了 MIL-101(Cr)的基本晶体结构, 但与 S2-MIL-101(Cr)的 XRD 谱图相比, 再生之后样品的 XRD 谱图的衍射峰强度有一定程度的下降, 表明再 生过程对 S2-MIL-101(Cr)的晶体结构产生了一定程 度的影响. 从图 2 中可以看出, 经过三次再生之后的 样品中仍然含有磺酸基团的四个特征吸收峰, 只是 吸收峰的强度明显降低, 表明磺酸基团在吸附和再 生过程中有所损耗. 因此, 对此吸附剂的再生方法 需要做进一步地探索.

\section{4 结 论}

通过与三氟甲磺酸䣶 $\left(\mathrm{Tf}_{2} \mathrm{O}\right)$ 和浓硫酸的磺化反 应, 对金属-有机骨架材料 MIL-101(Cr) 的配体进行 磺酸功能化修饰. 磺酸功能化后的 MIL-101(Cr)保持 了基本结构, 但结晶度、比表面积和孔径均有降低. 通过改变反应物之间的摩尔配比, 可以得到含有不 同磺酸基团数量的 MIL-101(Cr). 适度磺酸功能化能 够增强 MIL-101 $(\mathrm{Cr})$ 与含氮化合物的相互作用, 提高 其对含氮化合物的吸附容量, 并且更有利于对碱性 氮化物的吸附. 通过 Langmuir 吸附模型可以对磺化 后的样品吸附含氮化合物的等温线进行拟合. 拟合 结果表明: 按 MIL-101(Cr)、 $\mathrm{H}_{2} \mathrm{SO}_{4}$ 和 $\mathrm{Tf}_{2} \mathrm{O}$ 摩尔配比 为 1:3:4.5 反应得到的样品 S2-MIL-101 $(\mathrm{Cr})$ 对喹啉和 吲哚具有最大的 $Q_{0}$ 值, 相对于未经磺化的样品, $Q_{0}$ 分别提高了 $12.2 \%$ 和 $6.3 \%$. 经过三次再生, 该吸附剂 对含氮化合物的吸附量没有明显降低, 具有潜在的 应用前景.

\section{References}

(1) Furimsky, E.; Massoth, F. E. Catal. Today 1999, 52 (4), 381. doi: 10.1016/S0920-5861(99)00096-6

(2) Dong, D.; Jeong, S.; Massoth, F. E. Catal. Today 1997, 37 (3), 267. doi: 10.1016/S0920-5861(97)00022-9

(3) Xu, C. M.; Yang, Z. H. Petroleum Refining Engineering; Petroleum Industry Press: Beijing, 2009; pp 8-10［徐春明, 杨 朝合. 石油炼制工程. 北京: 石油工业出版社, 2009: 8-10]

(4) Schlatter, J. C.; Oyama, S. T.; Metcalfe Iii, J. E.; Lambert, J. M., Jr. Ind. Eng. Chem. Res. 1988, 27 (9), 1648. doi: 10.1021/ ie00081a014

(5) Choi, J.; Brenner, J. R.; Colling, C. W.; Demczyk, B. G.; Dunning, J. L.; Thompson, L. T. Catal. Today 1992, 15 (2), 201. doi: 10.1016/0920-5861(92)80176-N

(6) Song, H.; Guo, Y. T.; Li, F.; Yu, H. K. Acta Phys. -Chim. Sin. 2010, 26, 2461. [宋 华, 郭云涛, 李 锋, 于洪坤. 物理化学 学报, 2010, 26, 2461.] doi:10.3866/PKU.WHXB20100937

(7) Laredo, G. C.; Leyva, S.; Alvarez, R.; Mares, M. T.; Castillo, J.; Cano, J. L. Fuel 2002, 81 (10), 1341. doi: 10.1016/S0016-2361 (02)00047-9

(8) Almarri, M.; Ma, X.; Song, C. Energy Fuels 2009, 23 (8), 3940 doi: $10.1021 / \mathrm{ef} 900051 \mathrm{r}$

(9) Sun, B.; Li, G.; Wang, X. J. Nat. Gas Chem. 2010, 19 (5), 471. doi: 10.1016/S1003-9953(09)60115-8

(10) Kim, J. H.; Ma, X.; Zhou, A.; Song, C. Catal. Today 2006, 111 $(1-2), 74$.

(11) Wu, J.; Li, X.; Du, W.; Dong, C.; Li, L. J. Mater. Chem. 2007, 17 (21), 2233. doi: 10.1039/b612501h

(12) Zhang, H.; Li, G.; Jia, Y.; Liu, H. J. Chem. Eng. Data 2009, $55(1), 173$.

(13) Laredo, G. C.; Vega-Merino, P. M.; Trejo-Zárraga, F.; Castillo, J. Fuel Proces. Technol. 2013, 106, 21. doi: 10.1016/j. fuproc.2012.09.057

(14) Volkringer, C.; Loiseau, T.; Férey, G.; Morais, C. M.; Taulelle, F.; Montouillout, V.; Massiot, D. Microporous Mesoporous Mat. 2007, 105 (1-2), 111.

(15) Cychosz, K. A.; Wong-Foy, A. G.; Matzger, A. J. J. Am. Chem. Soc. 2008, 130 (22), 6938. doi: 10.1021/ja802121u

(16) Blanco-Brieva, G.; Campos-Martin, J. M.; Al-Zahrani, S. M.; Fierro, J. Fuel 2011, 90 (1), 190. doi: 10.1016/j.fuel.2010.08.008

(17) Khan, N. A.; Jhung, S. H. Fuel Process. Technol. 2012, 100, 49. doi: 10.1016/j.fuproc.2012.03.006

(18) Maes, M.; Trekels, M.; Boulhout, M.; Schouteden, S.; Vermoortele, F.; Alaerts, L.; Heurtaux, D.; Seo, Y.; Hwang, Y. K.; Chang, J.; Beurroies, I.; Denoyel, R.; Temst, K.; Vantomme, A.; Horcajada, P.; Serre, C.; De Vos, D. E. Angew. Chem. Int. Edit. 2011, 50 (18), 4210. doi: 10.1002/anie.v50.18

(19) Ahmed, I.; Hasan, Z.; Khan, N. A.; Jhung, S. H. Appl. Catal. B 2013, 129, 123. doi: 10.1016/j.apcatb.2012.09.020

(20) Wang, Z.; Cohen, S. M. Chem. Soc. Rev. 2009, 38 (5), 1315. doi: $10.1039 / \mathrm{b} 802258 \mathrm{p}$ 
(21) Férey, G.; Mellot-Draznieks, C.; Serre, C.; Millange, F.; Dutour, J.; Surblé, S.; Margiolaki, I. Science 2005, 309, 2040. doi: 10.1126/science. 1116275

(22) Goesten, M. G.; Juan-Alcañiz, J.; Ramos-Fernandez, E. V.; Sai Sankar Gupta, K. B.; Stavitski, E.; van Bekkum, H.; Gascon, J.; Kapteijn, F. J. Catal. 2011, 281 (1), 177. doi: 10.1016/j.jcat. 2011.04 .015

(23) Akiyama, G.; Matsuda, R.; Sato, H.; Takata, M.; Kitagawa,
S. Adv. Mater. 2011, 23 (29), 3294. doi: 10.1002/adma. 201101356

(24) Atorngitjawat, P.; Klein, R. J.; Runt, J. Macromolecules 2006, 39 (5), 1815. doi: 10.1021/ma051717f

(25) Warren, D. S.; Mcquillan, A. J. J. Phys. Chem. B 2008, 112 (34), 10535. doi: $10.1021 / j p 801838$ n

(26) Ostrowska-Gumkowska, B.; Ostrowska-Czubenko, J. Euro. Poly. J. 1994, 30 (8), 869. doi: 10.1016/0014-3057(94)90017-5

勘 误

倪哲明, 夏明玉, 施 炜, 钱萍萍. 糠醛在 Pt(111)表面的吸附和脱碳反应. 物理化学学报, 2013, 29 (9), 1916, 论文的中文题目 “糠醛在 $\operatorname{Pt}(111)$ 表面的吸附和脱碳反应”改为 “糠醛在 $\operatorname{Pt}(111)$ 表面的吸附和脱羰反 应”. TOC 和文章图 5 中糠醛的化学结构

\section{Corrigendum}

NI Zhe-Ming, XIA Ming-Yu, SHI Wei, QIAN Ping-Ping. Adsorption and Decarbonylation Reaction of Furfural on Pt(111) Surface. Acta Phys. -Chim. Sin. 2013, 29 (9), 1916, the Chinese title “糠醛在 Pt(111)表面的吸附 和脱碳反应” should be “糠醛在 Pt(111)表面的吸附和脱羰反应”, and the chemical structure of furfural

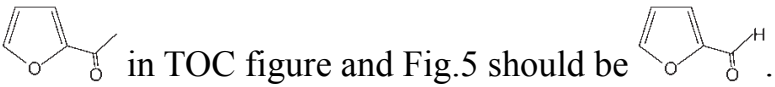

